\title{
Mathematical Representation: playing a role
}

\author{
Kate Hodesdon
}

May 31, 2013

\begin{abstract}
The primary justification for mathematical structuralism is its capacity to explain two observations about mathematical objects, typically natural numbers. Non-eliminative structuralism attributes these features to the particular ontology of mathematics. I argue that attributing the features to an ontology of structural objects conflicts with claims often made by structuralists to the effect that their structuralist theses are versions of Quine's ontological relativity or Putnam's internal realism. I describe and argue for an alternative explanation for these features which instead explains the attributes them to the mathematical practice of representing numbers using more concrete tokens, such as sets, strokes and so on. ${ }^{1}$
\end{abstract}

\section{Introduction}

Structuralism about mathematics is motivated by its capacity to provide an explanatory account of significant features of mathematics, especially the multiple realizability of mathematical objects which Benacerraf drew attention to in his article "What Numbers Could Not Be" (1965). It is on the basis of explaining these features that structuralism posits an ontology of structural objects. ${ }^{2}$ In this paper I examine the justification for structuralism's ontological move, and its compatibility with the claim often made by structuralists that their theories have precedents in the highly general responses

\footnotetext{
${ }^{1}$ I acknowledge the generous support of the Analysis Trust in carrying out this work. I am grateful to Brian King, who was the respondent to this paper at the sixth annual Graduate Conference on the Philosophy of Logic and Mathematics at Cambridge University, and to audiences at Bristol and Cambridge Universities.

Forthcoming in Philosophical Studies.

${ }^{2}$ For an example of the claim that structuralism is an explanatory account, see Resnik (1997, p.9; p.199).
} 
of Quine and Putnam to referential underdetermination. Then I describe an alternative way of explaining the phenomena explained by structuralism, which attributes them to mathematical practice, rather than to its subject matter.

In contrasting my account with structuralism, the theories that I will consider are: Shapiro's ante rem structuralism, expanded upon in (1997) and subsequent papers; Resnik's pattern theory, as described in (1981), (1996) and (1997); and Parsons' account, developed in (1990), (2004) and (2008). These are all non-eliminative versions of structuralism, which is to say that the accounts do not paraphrase away apparent reference to number, as they might, for instance, by taking arithmetic sentences to be covert universal quantifications over all appropriately structured domains as the in rebus structuralist account advocates.

These observations that structuralism seeks to explain are the following:

\section{(1) Multiple realizability: "anything at all can be the number 2"}

Mathematical objects are multiply realizable in the sense that formalized mathematical theories do not determine their objects; arrangements of objects drawn in whole or in part from distinct domains may serve as the objects of the theory, as long as they are intra-related in the appropriate way. There are therefore infinitely many accounts that tell us what the number 2 is, all of which take it to be something different. And so, as Benacerraf argued, if the number 2 is a particular object, then just which object it is is underdetermined by arithmetic. But, "the position that this is an unknowable truth is hardly tenable" (1965, p.62). Benacerraf was led to suggest a structuralist thesis in his conclusion that "number theory is the elaboration of the properties of all structures of the order-type of the numbers" (Ibid. p.71). This addresses the problem of multiple realizability by claiming that although the different set-theoretic reductions of arithmetic may appear to present us with a number of equally good candidates for being the numbers, none are in fact suitable: "numbers are not objects at all, because in giving the properties (that is, necessary and sufficient) of numbers you merely characterize an abstract structure" (Ibid. p.70).

Although intended speculatively, ${ }^{3}$ the Dedekindian spirit of Benacer-

\footnotetext{
${ }^{3}$ Benacerraf later clarified that his (1965) conclusion that numbers could not be objects
} 
raf's remarks has been firmly appropriated by mathematical structuralism. Mathematical structuralism is motivated as a way of accounting for the multiple realizability phenomenon Benacerraf described (Shapiro, 1997, p.78-81; Resnik 1997, p.267). The structuralist explanation is that the different settheoretic reductions of arithmetic are simply different ways of filling the places of the natural number structure. According to structuralism, it is the structure itself that is the subject-matter of arithmetic, and its positions, rather than the things in filling them, that are the mathematical objects. In this way, structuralism explains the apparent underdetermination of mathematical ontology that multiple realizability demonstrates by claiming that mathematical ontology is composed of structural positions, and not of sets, nor any other particular objects. ${ }^{4}$ In what follows, I describe an alternative way of understanding what it means for an $\omega$-sequence to "serve as" the natural numbers.

The second observation that structuralism justifies is that mathematical objects are incomplete:

\section{(2) Mathematical objects are incomplete}

Structuralists' claims that mathematical objects are incomplete focus on two distinct notions of incompleteness, which I will treat separately. The distinction is based on one made by Øystein Linnebo (2008).

\section{(2.a) Incompleteness as property-scarcity:}

Structuralists have called mathematical objects incomplete in the sense that they have no "intrinsic nature", or properties other than structural ones (Resnik 1997, p.211, 267; Shapiro, 1997, p.79). As stated, the claim requires some qualification, for even on the structuralist account, numbers have the non-structural properties of being abstract, of being my favourite number, and so on. The recognition that a qualification is needed was first pointed out

was "more contemplated than advanced" (Benacerraf, 1996, p.50). In naming his defense of that particular thesis a "way out", Benacerraf intended to also signify a radical departure from the paper's earlier sections "in the sense of 'far out"' (Ibid). Unfortunately the pun was lost on just about all of his readers.

${ }^{4}$ Structuralists take the claim that mathematical objects are structural to apply to some, if not all, mathematical theories. Shapiro reserves the term "structure" to describe the subject matter of what he calls "nonalgebraic" theories, which are roughly those that are categorical or have a natural, categorical second-order completion. (Shapiro, 1997, p.73, n.2) 
by Mark Balaguer (1999) in his review of Resnik (1997). MacBride (2005, pp.583-4) and Linnebo (2008) have since made the same objection. In his review, Balaguer notes Resnik's assurance that he did not intend to deny that mathematical objects have non-structural properties, although their nonstructural properties are merely "non-distinguishing" ones. Shapiro has also retracted unconditional claims that all of a mathematical object's properties are structural (2006, pp.121-31). Nonetheless, the idea behind this notion of incompleteness is still more or less captured by the claim that mathematical objects are comparable to 'points' or 'positions', which are placeholders in a structure. This heuristic has been a motivating idea for structuralism from the start; see, for instance, this early remark by Resnik,

In mathematics, I claim, we do not have objects with an 'internal' composition arranged in structures, we have only structures. The objects of mathematics [...] are structureless points or positions in structures. As positions in structures, they have no identity or features outside a structure. (1981, p.5)

\section{(2.b) Incompleteness as underdetermination of cross-structural identities:}

The second incompleteness claim that is sometimes given is articulated in terms of the underdetermination of cross-structural identities. The label of incompleteness in this case is due to Parsons (1990), but the idea is shared by other influential structuralists, Resnik and Shapiro (Resnik, 1997, p.90; Resnik 1996, p.83; Shapiro 1997, p.258). Thus, Resnik writes that,

Mathematical objects are incomplete in the sense that we have no answers within or without mathematics to questions of whether the objects one mathematical theory discusses are identical to those another treats; whether, for example, geometrical points are real numbers. (Resnik, 1997, p.90)

This claim can be seen as a less restricted version of the first incompleteness claim that structural objects have no non-structural or non point-like properties: it says that in particular they have no inter-structural properties.

\section{§1. Mathematical Representation}

I will not examine justifications of these two observations here, and will assume that they are good and worth preserving in an account of mathematics. Instead I address the extent to which the observations indicate that mathematics has a structural ontology. I argue that the multiple realizability and 
incompleteness observations are not indicative of any ontological feature of mathematical objects, and, in particular, they do not indicate an ontology of structures, patterns or structural universals. This is because the features are ubiquitous, and not characteristic of mathematical objects in particular. Their ubiquity is a good reason not to provide an explanation for the features which only applies to the mathematical domain - as does the explanation that takes the features to be characteristic of a structural ontology. My argument is not motivated by a prior prejudice against structures as an ontological category, nor by a conviction that, as Azzouni puts it, structuralism "makes the ontologically radical move of substituting structures for the traditional platonic objects as the subject matter of mathematics" (1994, p.8). Rather, my motivation is the principle that an explanation of some ubiquitous explananda which accounts for all cases in which the explananda occur is a better explanation than one which accounts for them in isolated cases only. In $\S 3$ I suggest an account of the mathematical practice of representation, which, I argue, does the same explanatory work as structuralism, but subsumes the treatment of mathematical objects with that of other abstract or concrete objects that can be represented. In doing so, I aim to show that it is possible to endorse the structuralist's good insights into mathematics, without being committed to a structuralist ontology.

When Benacerraf first posed the multiple realizability of numbers, in certain places he describes it as a problem of the underdetermination of reference for arithmetic terms:

We have two (infinitely many, really) accounts of the meaning of certain words ('number', 'one', 'seventeen', and so forth) each of which satisfies what appear to be necessary and sufficient conditions for a correct account. [...] Furthermore, in Fregean terminology, each account fixes the sense of the words whose analysis it provides. Each account must also, therefore, fix the reference of these expressions. Yet, as we have seen, one way in which these accounts differ is in the referents assigned to the terms under analysis. (1965, p.56)

Each of these accounts gives us a different set, class, sequence, and so on, as the number 2. Benacerraf is right that none of these things are what we are looking for when we ask what the numbers are. But the $\omega$-sequences are collections that, for the purpose of doing mathematics, we can take to stand in for the natural numbers. The claim I wish to defend here is that the various $\omega$-sequences suggested as candidates for being the natural 
numbers should be understood as different representations of them. The sequences represent the natural numbers by way of other token objects, like sets, and so the Zermelo set $\{\{\emptyset\}\}$ and the von Neumann set $\{\{\emptyset\}, \emptyset\}$ are not two different candidates for being the number 2, but rather are two different representations of that number. As Benacerraf says, given different $\omega$-sequences which serve as the natural numbers, "there seems to be little to choose among the accounts. Relative to our purposes [...] one will do as well as another, stylistic preferences aside." (1965, p.62) Representation is by no means unique - any representation of number may be used to do arithmetic with-and stylistic preferences are all we have to choose between them.

On the representation account, the claim that both $\{\{\emptyset\}\}$ and $\{\{\emptyset\}, \emptyset\}$ can be the number 2 should be read in the same way as the claim that many different people can be Lady Macbeth in a production of the play. In a given production of Macbeth, there is one person who "is" (who represents) the character Lady Macbeth, although it is true that anyone could have played that role. And in productions enacted across different places and times, many distinct people will be Lady Macbeth. The situation is analogous to the different ways of filling roles in social institutions or in organized team sports, such as the role of president of the USA or of the shortstop in a baseball match, that Shapiro uses to illustrate his conception of positions in ante rem structures. Both representing something in a model or replica, and fulfilling a function in a social institution or game may be ambiguously described as 'playing a role'. ${ }^{5}$ Structuralism understands $\{\{\emptyset\}\}$ to be a candidate for playing the role of 2 in the latter sense, and goes on to reify the ante rem structure relative to which the role is defined (just like a baseball team, or workplace structure). The numbers are then claimed to be the structure's places, or roles, as in this definition from Rynasiewicz:

Structuralism,as a doctrine in the philosophy of mathematics, stands in opposition to mathematical realism (Platonism) by denying that, e.g., numbers are anything more than structural roles in the models of number theory (1996, p.59).

My alternative suggestion is that $\{\{\emptyset\}\}$ be understood as playing the role of

\footnotetext{
${ }^{5}$ On occasions in which somebody plays a very generic role in a play, such as the role of the hero or narrator, she may be thought of as playing a role in both senses of the term; she plays the role of the character described in the script, and she also fulfills the function that her character has in the plot being performed-for instance, the function of providing the subject of the plot, if she is the heroine, or of conveying the story, if she is the narrator.
} 
2 in the former sense. So, $\{\{\emptyset\}\}$ plays the role of 2 in same sense as that in which an actor stands in for the character they are portraying. The relevant notion of representation is intended to be quite general, and certainly not limited to the representation of fictional characters in drama. Just about any thing can represent any other, although in a given situation there will be better or worse representations. What an object represents, if it represents anything at all, is conventional. This non-uniqueness of representation explains the multiple realizability of mathematical objects. But, the nonuniqueness is highly general, and does not depend on the particular nature of the thing being represented. Further details of the representation relation, and a positive argument that tells in favour of the account I suggest, are found in $\S 3$ and $\S 2$ respectively.

Given an alternative explanation of multiple realizability, it falls to descriptions of the incompleteness of the objects of mathematics to support the claim that those objects are structural positions. But the incompleteness observation alone cannot bear this weight, for incompleteness is ubiquitous. Recall that mathematical objects are said to be incomplete in the first sense delineated above if they are metaphysically 'thin', and lack a certain kind of property - an "intrinsic nature", non-structural properties, or properties which outrun those had by a point or node in a structure. It's not at all clear what it means for mathematical objects to lack an intrinsic nature. ${ }^{6}$ But if the incompleteness of mathematical objects means that they are incomplete with respect to non-structural properties, then this doesn't seem unique to mathematical objects. For, incompleteness with respect to some property or other applies to all abstract objects, and - although this is more contentious - to some concrete ones too. Just as abstract objects lack spatiotemporal properties, tables arguably lack mathematical properties, for tables are neither prime nor are they composite. Mathematical objects are said to be incomplete in the second of the two senses defined above if there is no answer to questions about the identification of positions from different structures. In this respect, mathematical objects are, again, like fictional characters, for there is no fact of the matter to some questions of identification between fictions. We simply don't have enough information to decide whether the witch from Hansel and Gretel is the same as the witch in Sleeping Beauty. It therefore appears that the incompleteness observation - in either of the senses described - is true of lots of different objects, and so, on

\footnotetext{
${ }^{6}$ For another argument that structuralists would do well to shift the focus of their view away from the incompleteness claim, see Linnebo (2008, pp.62-6).
} 
its own, it doesn't justify positing a distinct kind of ontology for mathematics.

\section{$\S 2$. Multiple realizability as referential underdetermination}

In the previous section, I suggested that multiple realizability could be understood as the result of the underdetermination of suitable representations of mathematical objects. If this is the case, then multiple realizability is a lot like the referential underdetermination demonstrated by arguments such as Quine's inscrutability thesis (1960, pp.51-79; 1968), or Putnam's modeltheoretic arguments (1981, Ch.2; 1980). Referential underdetermination arguments demonstrate that there is no unique permissible reference relation, and, in fact, that those permitted may be radically different. The arguments show that some given object, say a cat, can be denoted in discourse in many different ways, all of which preserve the truth of whichever theory we wish to preserve; it can be denoted by the name 'cat', the name 'cherry', and so on. In fact, referential underdetermination arguments usually proceed by fixing a particular name, and showing that, in a truth-preserving way, that name can be taken to denote many different objects - but this is equivalent to showing that one object can be referred to by different names. In just the same way, an object can be denoted in many different ways using different representative tokens. However, the underdetermination of representation should be far less striking than the conclusions of the Quine/Putnam theses, since it confronts us as soon as we think about how to go about representing. For instance, imagine that you need to describe to some travelers whereabouts a particular city is in your country: the easiest thing to do is to make a mock-up of the situation, perhaps by saying something like "if this glass is London, and the edge of the table is the south coast, then Brighton is roughly where that beer mat is". But, of course, swap the glass and the mat, and you have an arrangement that can represent the very same situation. In the same way, any one of the actors in a cast could have played Lady Macbeth; permuting people between roles will nevertheless result in a representation of the same events of Macbeth. The underdetermination is due simply to the combinatorial fact that there are many isomorphic, but extensionally distinct ways to model some phenomenon.

One aspect of mathematical structuralism that has been largely overlooked in the literature is its proponents' claim that their structuralist theories are versions of particular philosophical positions that are designed to

resolve referential underdetermination. Specifically, these are the problems 
of Quine's inscrutability argument, and Putnam's permutation argument, which is intended to generalize Quinian inscrutability by demonstrating radical underdetermination of reference. ${ }^{7}$ Resnik and Parsons have each claimed that their structuralist resolutions of multiple realizability are versions of ontological relativity, which is the theory Quine took to meet the challenges of inscrutability. Thus, Parsons states:

Clear general statements of the kind of view I have in mind [i.e., non-eliminative structuralism] were made by [...] W. V. Quine [...] in connection with his doctrine of 'ontological relativity'. (1990, p.304)

The same claim is made by Resnik, who emphasizes the Quinian heritage of his structuralist thesis:

Quine has captured the incompleteness of mathematical objects in his doctrine of ontological relativity. [...] What I have tried to do so far is show that Quine's surprising doctrine is what we would expect to hold in mathematics. (1996, p.86; See also 1997)

Shapiro claims that ante rem structuralism is a version of Putnam's internal realism, where internal realism is the position that Putnam took to be immune to referential underdetermination arguments:

If I have accurately interpreted Putnam's suggestive remarks, then in mathematics at least, ante rem structuralism is a version of the (1987) incarnation of internal realism. (1997, p.128; see also pp.65-7)

I take it that the idea behind these likeness claims is something like the following. Multiple realizability entails that the Zermelo 2 and the Von Neumann 2 are equally good candidates for being the number two. Therefore, both of these objects are equally good candidates for the name ' 2 ' to refer to. This means that the reference of ' 2 ' is underdetermined between these objects. Quine's ontological relativity and Putnam's internal realism were both devised to be immune to problems of underdetermination of reference. And, like these theories, structuralism also gives a resolution to the underdetermination of the reference of ' 2 '.

\footnotetext{
${ }^{7}$ For Putnam's claim that the permutation argument generalizes Quine's argument for the inscrutability of reference, see (1981, p.35).
} 
Given Benacerraf's presentation of the multiple realizability of numbers as a problem concerning the underdetermination of reference of arithmetic language, Crispin Wright raised the following objection to Benacerraf's problem in Frege's Conception of Numbers as Objects. Wright acknowledged that the problem Benacerraf identifies for numerical singular terms - that is, the problem that they have many equally good candidate referents - extends quite generally to the problem that there are many possible candidates for the reference of any singular term. But this generalization of Benacerraf's problem treads "familiar ground", covered by Quine in his Gavagai argument. Wright's charge is that Benacerraf hasn't succeeded in raising any new problem that tells against mathematical platonism in particular (1983, pp.117-129).

I want to raise a related problem for the claim that mathematical structuralism is a version of ontological relativity or Putnam's internal realism. The problem is that the claim is in tension with structuralism's positing a particular kind of ontology for mathematical domains. Quine and Putnam's indeterminacy arguments are fully general by design; they're not domain specific, so they apply to mathematical domains by default. ${ }^{8}$ Quine explicitly discusses the extension of ontological relativity to mathematical domains of the kind Benacerraf described. ${ }^{9}$ And so, if structuralism is a version of one of the positions resolving the referential indeterminacy - as its proponents claim it is - then structuralists owe an explanation for why

\footnotetext{
${ }^{8}$ Not only is Putnam's argument not domain-specific, it is also independent of the choice of language. Putnam points out, in response to criticism that his argument from the Löwenheim-Skolem theorem is a uniquely first-order problem, that another of his model-theoretic arguments, the permutation argument, uses "a technique - permutation of individuals - which applies to second-order logic, modal logic, tensed logic, etc" (Putnam, 1995, p.356, n.11).

${ }^{9}$ See Quine's remarks in "Ontological Relativity":

Numbers in turn are known only their laws, the laws of arithmetic, so that any constructs obeying those laws - certain sets, for instance-are eligible in turn as explications of number. Sets in turn are known only by their laws, the laws of set theory.

$[\ldots]$

[A]ny progression will serve as a version of number so long and only so long as we stick to one and the same progression, Arithmetic is, in this sense, all there is to number: there is no saying absolutely what the numbers are; there is only arithmetic. (1968, p.44-5)
} 
their resolution - which is to posit an ontology of structural objects - is only a solution that goes for mathematical objects, and not for concrete objects like cats and cherries. Of course, the structuralist could posit a global structuralism, which takes in non-mathematical and mathematical objects alike. Shapiro discusses the prospects for extending the structuralist account to treat objects beyond the mathematical in Chapter 8 of (1997). This would prevent my "inconsistent approach" objection that mathematical structuralism responds to mathematical referential underdetermination in a way that is at odds with responding to the referential underdetermination of our everyday language. However, in so far as mathematical structuralism responds to the underdetermination by positing a structural ontology for mathematical objects only (and in Shapiro's case, he applies this just to a specific category of mathematical object - see footnote 3 above), structuralism just cannot be a version of Putnam's internal realism, nor of Quine's ontological relativity.

If reference is a special case of representation, then my proposal that we understand multiple realizability to be due to the existence of many representations of number would constitute an extension of the Quine/Putnam referential underdetermination problems to the mathematical domain. It would therefore vindicate the claims shared by Parsons, Resnik and Shapiro about the similarity between structuralism, as a response to this problem, and the Quine/Putnam responses to referential underdetermination. But the proposal also avoids the worry that multiple realizability picks out too general a phenomenon for it to warrant a resolution which applies to mathematical domains only.

\section{$\S 3$. Structuralism's Ontological Commitments}

It may be objected that structuralism doesn't just answer the demand for an explanation of the multiple realizability and incompleteness observations. Some structuralist accounts do more than this, and also tell us what the proper subject matter of mathematics is: namely, structures, or structural positions. Thus, Shapiro (1997, p.5) tells us that "[t]he subject matter of arithmetic is the natural-number structure, the pattern common to any system of objects that has a distinguished initial object and a successor relation that satisfies the induction principle." And, more generally "the subject matter of [a mathematical] theory is [a] structure or class of structures." (Ibid. p.13) In Shapiro's account, this makes the subject matter of a mathematical theory a certain class of universals (2008, p.302). Parsons' structuralist 
account instead claims that the objects of mathematics are structural positions, not the structures themselves (2008, p.111).

Clearly my account does not touch upon the question of the proper ontology for mathematics. My claim is that concrete $\omega$-sequences are related to the natural numbers by a representation relation, and that this representation explains the structuralist claim that "anything at all can 'be' 2" (Shapiro 1997, p.80). But, evidently, to say that the Zermelo ordinals represent the natural numbers raises the question of just what the numbers being represented are. On this matter, my account is neutral. The mathematical practice of representation which I have sketched here is compatible both with platonist and with nominalist answers. Its consistency with nominalism depends on whether or not it is possible to represent non-existent objects. On this, I will say just that I take it that there are examples instantiating just this possibility: paintings of Pegasus, for example.

So, unlike Shapiro and Parsons' structuralist theories, my account does not claim to tell us the right ontology for mathematics. Does this mean that my account fails to address a question that structuralism gives us an answer to? Well, not quite; because not all structuralist accounts of mathematics tell us what the proper ontology of mathematics is. Resnik describes how his slogan that "mathematical objects are positions in patterns" is best interpreted with an epistemic reading, and he distances himself from claiming that there is an ontology of structures, or of structural places. The epistemic reading Resnik has in mind is based on a passage of Quine, in which Quine describes how his claim that theories determine only structures should not be taken as suggesting that the entities those theories treat are structural objects. Rather, Quine claims, the thesis should be understood as belonging to the "methodology of ontology, and thus to epistemology" (1981, p.21-2). Resnik intends that his structuralist thesis be understood in the same way. Resnik's epistemic move is little-discussed in the literature on structuralism, and invites comparisons with the structuralist position in the philosophy of science in which Ladyman has drawn a distinction between ontic and epistemic versions of structural realism (1998).

Resnik denies that mathematical objects are literally structural positions (1981, pp.269-70). As a result, his structuralism should not be seen as providing an ontological reduction of number, where a programme of ontological reduction gives us the kind of thing that Frege sought for the natural numbers, and Dedekind for the reals. Resnik explains that, 
I have been taking yet another approach to the question of what mathematical objects are [...] My suggestion that mathematical objects are positions in patterns is not intended as an ontological reduction. My intention was instead to offer another way of viewing numbers and number theory which would put the phenomenon of multiple reductions and ontological and referential relativity in a clearer light. (1997, p.223)

The representational account of mathematics I've sketched here is, likewise, intended as a way of explaining multiple realizability, without positing an(other) ontological reduction for mathematical objects.

\section{$\S 4$. What is the representation relation?}

Having motivated the representational account, I'll fill in some more details about what I mean by a representation relation. I follow van Fraassen (2008) in taking representation to be a ternary relation; a subject $x$ is represented by an agent $y$, using some object $z$. The way that we represent numbers has partial analogues in the representation of actual or possible objects. For example, a cartographer might represent the landscape of a country via a map. In a similar way, I can use pieces of paper arranged on a table to represent a possible seating plan. The pieces of paper needn't be inscriptions of the names of the people that they represent: it suffices for their being representations that I intend to use them in this way, and that anyone with whom I share my representation is aware of my intentions. It also seems plausible that, in order to successfully represent something, I know that my audience is aware of my intentions, and that they know that I am aware of this, etc., in accordance with Grice's conditions for implicature (1989, pp.22-40).

In the examples given above of the map and the seating plan the audience's attention is directed to a representation of the subject matter precisely because the subject itself cannot be directly ostended. We use maps to understand the salient features of a country because we cannot actually see those features from any accessible vantage point; and a representation of a seating arrangement is useful because I cannot point to the hypothetical arrangement of people. In each of these cases, the subject represented could have been described in words, instead of represented with non-linguistic items like contour lines or bits of paper. In this case, the words would have referred to the landscape, or to the people. I take reference to be a special 
case of representation relation, but it would be possible to endorse an account of the kind described here if, instead, reference were to be understood as a distinct, but relevantly similar, intentional relation. The reasons why we might use a (nonlinguistic) representation of a subject rather than a linguistic description of it overlap with the reasons why modeling has pragmatic value in science.

In the two examples of representation given above, the things represented are concrete objects - people and places - which have features in addition to those that they are represented as having: for instance, the people have heights and ages that are not represented my pattern of name places. In making the representation, only the salient properties of the subjects are portrayed, where what is salient depends on the purpose of the representation. Unlike these examples, the representation of the natural numbers by way of the Zermelo ordinals portrays all of the numbers' mathematical properties. The Zermelo ordinals have all the mathematical properties that the natural numbers do: they have the right order properties, and admit definition of the arithmetic operations. But mathematical representation is not in principle unlike representation in other contexts. The reason why representations of mathematical objects typically respect all of the objects' mathematical properties is that, typically, all of the properties will be salient to the use of the representation. Using a representation of the natural numbers for the purpose of doing arithmetic aims at discovering new facts, or at proving already-known ones, about numbers. Our chance of fulfilling this aim is maximized if we use a representation which is maximally accurate in the sense that it respects as many truths as possible.

It might be inferred from instances of mathematical representation which $d o$ respect all of their subject's properties that all mathematical representation is extensional: that, for example, to represent a number as being the third natural number is to represent it as being the first prime. But I do not want to claim this. Van Fraassen has argued that to represent something as such-and-such is intensional (2008, pp.26-8). For instance, we can represent a creature as having a heart without representing it as having kidneys. I see no reason why mathematical representation should differ. It is true that examples of non-extensional representations of mathematical objects are harder to come by than examples involving other kinds of object. Nevertheless, I maintain that mathematical representation is intensional; the reason why examples of non-extensional representations of number are hard to come across is that, as claimed above, for the purpose of doing math- 
ematics a representation of number that doesn't lose any of the numbers' properties is just a superior representation.

Mathematical representation is not the only example in which it is possible to represent all of a subject's properties. It is also possible to represent all of the properties of a fictional character, as, for example, in an especially faithful reproduction of a play. To return to the example of the fictional character Lady Macbeth, she has no more properties, qua fictional character, than those that she is represented as having by an actor who sticks rigidly to the script in a performance of the play. However, while it is possible to represent all of the properties of a fictional character in this way, since the purpose of a play encourages actors to vary and re-imagine the properties of the characters they depict, such a faithful performance will not be a typical one. ${ }^{10}$

\section{$\S 5$. Conclusion}

To summarize: in opposition to structuralism, I deny that the multiple realizability phenomenon that Benacerraf described in his 1965 article tells us anything about what numbers are. Rather, multiple realizability reflects a fact about mathematicians' practice of representing the natural numbers, in most cases, within set theory. As well as being an explanatory account for multiple realizability, one advantage of the representation account is that it brings the treatment of mathematical objects closer in line with the way that we treat other abstract entities that we cannot point to, such as fictional characters and non-actual objects (recall the hypothetical seating plan); we treat them via representations.

So, what it takes for a sequence to "be" the natural numbers is different on my account. What is important about the Zermelo ordinals and the von Neumann ordinals is not just that they have the right structure, or instantiate the right structural universal; instead, it's that they're used to do arithmetic with. Perhaps instantiating the right structure is a necessary condition for objects to represent the natural numbers. However, instantiating the right structure can't be a sufficient condition for representation, and hence the two notions come apart, because representation is a matter of intention.

\footnotetext{
${ }^{10} \mathrm{I}$ am grateful to an anonymous referee for emphasizing the disanalogy between mathematical representation and the representation of fictional objects in this respect.
} 
I'll conclude by drawing a parallel with a structuralist position recently defended in the philosophy of physics. Van Fraassen's empiricist structuralism (2008) likewise attributes structuralist claims to the practice of representation in science. The philosophy of physics has witnessed the development of a huge variety of structuralist accounts whose target is the physical world. Structuralist theses about a physical domain claim that all that we can know about that domain is its structure. Some structuralist theses go further, and add to the epistemic claim the ontic claim that all there is to the domain is structure. Van Fraassen interprets the core epistemic claim of physical structuralism by first adopting an empiricist view of scientific theories, and then arguing that "all we know through science is structure" (2008, p.238). He argues that what is preserved by successive theories is their adequacy to the phenomena, and that the phenomena are described by laws in the form of mathematical equations. However, mathematical equations can only describe anything up to isomorphism (1989), and so, only up to structural equivalence. Van Fraassen's empiricist structuralism therefore attributes the structuralist claim that science only deals with structure to the use of mathematics to represent physical phenomena.

Since, for Van Fraassen, the reason we can only describe the structure of the world originates with our representational practice, and its limitations, empiricist structuralism is a view about science, not an ontological theory about the world. He writes of empiricist structuralism that

$[\mathrm{T}]$ he formulation [does not] imply that there is in nature or in the phenomena a form/content or a structure/quality distinction to be drawn. The structuralism in "empiricist structuralism" refers solely to the thesis that all scientific representation is at heart mathematical. Empiricist structuralism is a view not of what nature is like, but of what science is. (2008, pp.238-9)

My proposal for mathematics parallels Van Fraassen's for science. I attribute the structuralist phenomenon of multiple realizability not to the nature of mathematical objects, but instead to the activity of mathematics. 


\section{References}

Azzouni, J.: Metaphysical Myths, Mathematical Practice: the Ontology and Epistemology of the Exact Sciences. Cambridge University Press, 1994

Balaguer, M.: Critical Study: Michael Resnik, Mathematics as a Science of Patterns. Philosophia Mathematica, 7 1999, Nr. 1, 108-126

Benacerraf, P.: What Numbers Could Not Be. The Philosophical Review, 74 1965, Nr. 1, 47-73

Benacerraf, P.: What mathematical truth could not be. 1996, 33-77

Grice, P.: Studies in the Way of Words. Harvard University Press, 1989

Ladyman, J.: What is Structural Realism? Studies in History \& Philosophy of Science, 29 1998, Nr. 3, 409-424

Linnebo, Ø.: Structuralism and the Notion of Dependence. The Philosophical Quarterly, 58 2008, Nr. 230, 59-79

MacBride, Fraser: Structuralism reconsidered. 2005, 563-589

Parsons, C.: The Structuralist View of Mathematical Objects. Synthese, 84 1990, Nr. 3, 303-346

Parsons, C.: Mathematical Thought and Its Objects. Cambridge University Press, 2008

Parsons, Charles: Structuralism and Metaphysics. The Philosophical Quarterly, 54 2004, Nr. 214, 56-77

Putnam, H.: Models and Reality. The Journal of Symbolic Logic, 45 1980, Nr. 3, 464-482

Putnam, H.: Reason, Truth, and History. Cambridge University Press, 1981

Putnam, H.: The Many Faces of Realism: The Paul Carus Lectures. La Salle, 1987

Putnam, H.; Conant, J., editor: Words and Life. Harvard University Press, 1995 
Quine, W.V.O.: Word and Object. Volume 1, M.I.T. Press, 1960

Quine, W.V.O.: Ontological Relativity. The Journal of Philosophy, 65 1968, Nr. 7, 185-212

Quine, W.V.O.: Theories and Things. Harvard University Press, 1981, Originally published in Journal of Philosophy, (1976), 73

Resnik, M.: Mathematics as a Science of Patterns: Ontology and Reference. Noûs, 15 1981, Nr. 4, pp. 529-550

Resnik, M.: Structural Relativity. Philosophia Mathematica, 4 1996, Nr. 2, 83-99

Resnik, M. D.: Mathematics as a Science of Patterns. Oxford University Press, 1997

Rynasiewicz, R.: Is There a Syntactic Solution to the Hole Problem? Philosophy of Science, 63 1996, pp. S55-S62

Shapiro, S.: Philosophy of Mathematics: Structure and Ontology. Oxford University Press, USA, 1997

Shapiro, S.: Structure and Identity. 2006, 109-145

Shapiro, S.: Identity, Indiscernibility, and ante rem Structuralism: The tale of i and -i. Philosophia Mathematica, 16 2008, Nr. 3, 285-309

Van Fraassen, B.C.: Laws and Symmetry. Oxford University Press, USA, 1989

Van Fraassen, B.C.: Scientific Representation: Paradoxes of Perspective. Oxford University Press, USA, 2008

Wright, C.: Frege's Conception of Numbers as Objects. Aberdeen University Press, 1983 